\title{
Randomised controlled trial of an online mother-daughter body image and well-being intervention
}

\author{
Phillippa C. Diedrichs, Melissa J. Atkinson, Kirsty M. Garbett, \\ Heidi Williamson, Emma Halliwell, Nichola Rumsey \\ Centre for Appearance Research, University of the West of England, United Kingdom
}

George Leckie

Centre for Multilevel Modelling, University of Bristol, United Kingdom

Chris G. Sibley

School of Psychology, University of Auckland, New Zealand

Fiona Kate Barlow

Menzies Health Institute Queensland, Griffith University, Australia

Corresponding Author: Phillippa Diedrichs, Centre for Appearance Research, University of the West of England, Coldharbour Lane, Bristol, BS16 1QY, UK;

phillippa.diedrichs@uwe.ac.uk; phone: +441173283756.

Declaration of Interest: This study was funded by a research grant from the Dove SelfEsteem Project (DSEP). The funders had no role in the study design, data collection and analysis, decision to publish, or manuscript preparation. They were permitted to review the manuscript, but the authors exclusively retained the final decision on content. PD is an independent advisor on the DSEP Global Advisory Board and receives a financial honorarium for this role. The authors declare no other potential conflicts of interest.

Citation: Diedrichs, P. C., Atkinson, M. J., Garbett, K. M., Williamson, H., Halliwell, E., Rumsey, N., Leckie, G., Sibley, C. G., \& Barlow, F. K. (2016, May 12). Randomized Controlled Trial of an Online Mother-Daughter Body Image and Well-Being Intervention. Health Psychology. Advance online publication. http://dx.doi.org/10.1037/hea0000361 


\begin{abstract}
Objective: Poor body image is a public health issue. Mothers are a key influence on adolescent girls' body image. This study evaluated an accessible, scalable, low-intensity internet-based intervention delivered to mothers (Dove Self-Esteem Project Website for Parents) on mothers' and their adolescent daughters' body image and psychosocial wellbeing. Methods: British mother-daughter dyads $(N=235)$ participated in a cluster randomized controlled trial [assessment-only control; mothers viewed the website without structured guidance (website-unstructured); mothers viewed the website via a tailored pathway (website-tailored)]. Dyads completed standardized self-report measures of body image, related risk factors, and psychosocial outcomes at baseline, 2-weeks post-exposure, 6-week and 12-month follow-up. Results: Dyadic models showed that relative to the control, mothers who viewed the website reported significantly higher self-esteem at post-exposure (website-tailored), higher weight esteem at 6-week follow-up (website-tailored), lower negative affect at 12-month follow-up (website-tailored), engaged in more self-reported conversations with their daughters about body image at post-exposure and 6-week follow-up, and were 3-4.66 times more likely to report seeking additional support for body image issues at post-exposure (website-tailored), 6-week, and 12-month (website-tailored) follow-up. Daughters whose mothers viewed the website had higher self-esteem and reduced negative affect at 6-week follow-up. There were no differences on daughters' body image, and risk factors among mothers or daughters, at post-exposure or follow-up. Tailoring website content appeared beneficial. Conclusions: This intervention offers a promising 'first-step' towards improving psychosocial well-being among mothers and daughters. In order to further optimise the intervention, future research to improve body image-related outcomes and to understand mechanisms for change would be beneficial.
\end{abstract}

Keywords: body image, self-esteem, intervention, internet, parents, adolescent girls. 
Poor body image is a public health issue, with governments and researchers calling for the dissemination of effective interventions (Paxton, 2015). Body dissatisfaction prospectively predicts depression and low self-esteem during adolescence, and is the most potent modifiable risk factor for eating disorders (Jacobi \& Fittig, 2010; Neumark-Sztainer, Paxton, Hannan, Haines, \& Story, 2006). Across Westernised countries, 34-62\% of girls experience poor body image (Al Sabbah et al., 2009). Despite an identified need, few body image interventions have been disseminated at scale (Stice, Becker, \& Yokum, 2013). As with most mental health interventions, this is likely due to multiple factors including an overreliance on face-to-face interventions and delivery by expert providers, participant barriers (e.g., time, scheduling, cost, perceived stigma), and a lack of funding and infrastructure for dissemination (Atkinson \& Wade, 2013; Patel, Kieling, Maulik, \& Divan, 2013). Consequently, there is an on-going need to develop and evaluate effective, acceptable, and scalable body image interventions.

Parents are an important influence on adolescent girls' body image. This is evidenced by a review of 56 cross-sectional and longitudinal studies conducted in North America, Australia, New Zealand, and Europe that examined the relationship between parents' attitudes and behaviours and their children's body image (Rodgers \& Chabrol, 2009). This review found that pressure to lose weight, appearance-related criticism, and modelling of body image concerns and dieting behaviours from parents are correlated with, and prospectively predict, poor body image among adolescent girls. Addressing parental influence on body image, therefore, offers a promising avenue for intervention. Importantly, interventions targeting parents can harness the powerful protective role parents can play by equipping them with tools to use with their children to foster positive body image. This could include helping their children to develop media literacy in order to resist appearance-related sociocultural pressures, and focusing on non-appearance related attributes and achievements 
(Hart, Cornell, Damiano, \& Paxton, 2014). Such interventions also provide an opportunity to support parents to develop their own positive body image, so that they are better able to role model positive body image behaviours and attitudes to their children.

To date, few body image interventions for children have targeted parents and been evaluated rigorously. A systematic review identified 20 studies conducted between 19922013 that evaluated interventions aiming to improve body image and/or prevent eating disorders among children and included an intervention component delivered to parents (Hart et al., 2014). Only three of these studies were sufficiently methodologically rigorous to determine reliable intervention effects on body image as a result of parental involvement. Of these, two interventions significantly improved adolescent girls' body image; one involved face-to-face workshops delivered to mothers (Corning, Gondoli, Bucchianeri, \& Salafia, 2010), and another consisted of an internet-based intervention for girls with a psychoeducational paper-based pamphlet sent to parents (Sniezek, 2006). The review noted small parent sample sizes were a common limitation across the studies reviewed, and few interventions were designed to meet the needs of parents. This suggests that there is a need to develop and rigorously evaluate body image interventions targeting parents.

As research suggests it is difficult to engage parents, it is important that interventions are designed to overcome barriers to uptake. Parents report a lack of time, scheduling conflicts, and financial costs as barriers to accessing parent-child mental health interventions (Ingoldsby, 2010). Internet-based interventions may overcome these barriers as they can be accessed anywhere and at any time, and they can be easily tailored to meet users' specific needs, which emerging research suggests can enhance intervention uptake and effectiveness (Bennett \& Glasgow, 2009). Additionally, it has been suggested that internet-based interventions are amenable to broad scale, cost-effective dissemination when they are program-led and not reliant on therapists (Fairburn \& Patel, 2014). Of course, internet-based 
interventions are not the only solution to addressing intervention scalability issues. Taskshifting to less expert providers has been used to successfully increase scalability of some face-to-face body image interventions (Kilpela et al., 2014). Nevertheless, several online body image interventions exist and have shown promise among adolescent girls and young adult women (e.g., Taylor et al., 2006). To our knowledge, however, there are no rigorously evaluated internet-based interventions specifically designed for parents to help them support their children to develop positive body image. This presents a significant gap in the suite of effective scalable interventions available to improve adolescent girls' body image.

This study aimed to evaluate the impact of a low-intensity internet-based body image intervention for parents (Dove Self-Esteem Project Website for Parents) among motherdaughter dyads. The website is designed to help mothers foster positive body image and psychosocial well-being among their daughters. Recognising that mothers' body image can influence their daughters', the website also aims to support mothers to develop their own positive body image. The website offers a scalable and sustainable intervention option. It is available at no cost in eight languages across the UK, Netherlands, France, Germany, Italy, US, Canada, Brazil, Argentina, Mexico, South Africa, and Australia, and it currently receives an average of 66,000 visits each month. The website is program-led, and has a sustainable funding model through the social mission agenda for the multi-national brand Dove. This intervention could therefore provide an accessible and convenient 'first step' towards promoting positive body image among adolescent girls and their mothers.

Previous research has found that internet-based interventions are likely to be most effective and acceptable when tailored (Bennett \& Glasgow, 2009). Therefore, this study also aimed to determine if providing mothers with a tailored pathway through the website could strengthen intervention effects. We hypothesized that relative to an assessment-only control group, mothers who viewed the website would experience improved body image, reduced 
severity of associated risk factors, improved related psychosocial outcomes, and increased help-seeking behaviors related to body image, as would their daughters. We also hypothesized that intervention effects would be stronger when mothers received a tailored pathway through the website, as opposed to browsing the website without structured guidance.

\section{Method}

\section{Trial Design}

A cluster randomised controlled trial was conducted with mother-daughter dyads across three regions in England (North, Midlands, South). Within each region, three towns were randomised using the random allocation rule to one of three experimental conditions (assessment-only control, website-unstructured, website-tailored). The trial was registered (ISRCTN57308485) and approved by the university ethics review board.

\section{Participants}

Mother-daughter dyads were recruited from nine towns in England by a market research agency to participate in a "website development study". Recruitment methods included telephone database sampling and approaching women face-to-face in shopping areas. Inclusion criteria were: daughter aged 11-14; dyads spoke English and used the internet more than once a week; mother identified body image as a relevant issue for their daughter; mother had participated in $\leq 3$ market research studies in their lifetime.

Participants were blind to study objectives and hypotheses, and were randomised to conditions by the market research agency. On completion, mothers and daughters were paid $£ 120$ and $£ 50$, respectively.

Sample size was calculated on the basis of detecting statistical significance of medium effect sizes (Cohen's $d=0.5$ ). This was adjusted for a medium degree of nonindependence $(r=0.3)$, resulting in an adjusted $d=0.4$ used for sample size calculations 
(Kenny, Kashy, \& Cook, 2006). Setting power at 0.8 and assuming a correlation between repeated measures of 0.5 , the required sample size was 74 dyads per condition (Twisk, 2006).

\section{Intervention}

The Dove Self-Esteem Project Website for Parents (selfesteem.dove.com) is an online information hub designed to provide mothers with information and tools to help them foster positive body image among their daughters and themselves. The website presents brief articles outlining expert advice, interactive activities (e.g., videos, games), and tips to encourage behaviour change. Professional writers in collaboration with body image researchers, psychologists, and youth workers developed the content. The content is evidence-informed and addresses five content themes based on empirically supported risk factors for poor body image; 1) Appearance-ideal media (e.g., developing resilience to unrealistic appearance ideals in media); 2) Appearance conversations (e.g., exploring how conversations about appearance, including "fat talk", can negatively influence body image); 3) Appearance-related teasing (e.g., exploring the impact of teasing and developing strategies to promote resilience); 4) Modelling and interpersonal relationships (e.g., exploring how family and friends can model body image behaviours and attitudes); and 5) Respecting and looking after yourself (e.g., body acceptance activities).

This study evaluated the live website in June 2014. In the website-unstructured condition, mothers browsed the website without structure or guidance. In the website-tailored condition, mothers were provided with a written personalised pathway that guided them to view two articles describing how they can develop their own positive body image, six articles matching the two content themes they identified as being most relevant to them and their daughters via the tailoring measure below, and 2-4 optional articles to view at home that corresponded to their preferred themes. These pathways were prepared in advance by the researchers, whereby the researchers selected articles on the website that best matched each 
of the corresponding risk factor content themes.

\section{Measures}

Participant characteristics. Self-reported demographics included age, country of birth, mother's marital status, ethnicity, and household chief wage earner's occupation. The latter was collected from mothers only and was categorised into social grade classifications in accordance with the UK National Readership Survey (2015). Body mass index (BMI; $\mathrm{kg} / \mathrm{m}^{2}$ ) was calculated using objective height and weight measurements taken by researchers at baseline.

\section{Intervention outcomes.}

Primary outcome. Body image. The Appearance Esteem (10 items) and Weight Esteem (8 items) subscales of the Body Esteem Scale for Adults and Adolescents (Mendelson, White, \& Mendelson, 1997) were used to assess body image. Subscale items assess general feelings and satisfaction regarding appearance (e.g., "I worry about the way I look”) and weight (e.g., "I am satisfied with my weight”) respectively using a 5-point Likert scale (never to always). A mean score was calculated for each subscale separately, with higher scores indicating greater body esteem. Scale reliability and validity has been previously demonstrated in adults and adolescents (Mendelson, Mendelson, \& White, 2001). Internal consistency in the current study was good for appearance esteem (Cronbach's $\alpha=.88$ for both mothers and daughters) and weight esteem (Cronbach's $\alpha=.90$ and .92 for mothers and daughters, respectively).

Secondary outcomes. Risk factors for poor body image. Internalisation of sociocultural appearance ideals was assessed using the General Internalisation subscale of the Sociocultural Attitudes Towards Appearance Scale-3 (SATAQ-3; Thompson, van den Berg, Roehrig, Guarda, \& Heinberg, 2004). This scale contains 8 items (e.g., "I would like my body to look like the bodies of people who are on $T V$ '). Responses are rated on a 5-point scale 
(definitely disagree to definitely agree), with higher scores indicating greater internalisation. This scale has demonstrated reliability and validity, and is widely used, among adults and adolescents (Thompson et al., 2004). Internal consistency in the current study was good (Cronbach's $\alpha=.95$ and .94 for mothers and daughters, respectively).

Perceived sociocultural appearance pressures were assessed using an adaptation of existing validated measures: the Pressures subscale of the SATAQ-3 (Thompson et al., 2004); and the Perceived Sociocultural Pressures Scale (9 items related to pressure from family, friends, partners, media; Stice \& Bearman, 2001). The resulting measure contained 12 items assessing perceived pressure to change aspects of appearance across four categories (i.e., "I have felt pressure to 'lose weight'/ 'change my body shape/build'/ 'have bigger muscles'/ 'change my appearance"') from three sources (family, friends, and media). Responses were rated on a 5-point scale (none to a lot), with higher scores reflecting greater perceived pressure. Internal consistency in the current study was good (Cronbach's $\alpha=.86$ and .84 for mothers and daughters, respectively).

Appearance-related social comparisons was assessed with the previously validated Social Comparison to Models and Peers Scale (Jones, 2001), which was expanded to include 8-items measuring the tendency to make comparisons with respect to four categories of 'weight', 'body shape/build', 'face', and' fashion/style', to both 'celebrities and people in the media' and 'other people my age'. Frequency of making comparisons was rated on a 5-point scale (never to $a$ lot), with higher scores reflecting a greater tendency to make comparisons. Internal consistency in the current study was good (Cronbach's $\alpha=.90$ and .91 for mothers and daughters, respectively).

Distress as a result of appearance-related teasing was assessed among daughters only, using teasing items validated in Project Eat-III (Neumark-Sztainer et al., 2007), which were adapted to assess feelings of upset about teasing regarding 'weight and shape', and 'the way 
you look' (2 items). Distress was scored on a 5-point scale (not upset to very upset), with higher scores reflecting greater distress. Internal consistency among daughters in the current study was good (Cronbach's $\alpha=.76)$.

Appearance-based conversations were assessed using the Appearance Conversations with Friends subscale of Culture Among Friends (Jones, Vigfusdottir, \& Lee, 2004). It has 5 items assessing the frequency of engaging peers in conversation around appearance (e.g., "my friends and I talk about what we would like our bodies to look like"), assessed on a 5point scale (never to very often). Although previously demonstrated to be reliable with adolescents (Jones et al., 2004), to our knowledge this is the first use among adults. Good internal consistency was demonstrated in the current study (Cronbach's $\alpha=.91$ and .91 for mothers and daughters, respectively).

Dyadic factors. The frequency of mother-daughter conversations about body image was assessed using a single-item ("How often do you speak to your mother/daughter about body image?"). This was rated on a 5-point scale (never to very often), with higher scores reflecting a greater frequency of body image conversations. In addition to conversations, daughters' perception of appearance-related pressures from their mother was assessed among daughters only using the validated Maternal Pressures Scale (Corning et al., 2010). It assesses maternal perceived pressure related to weight and shape, and was expanded to include additional items assessing general appearance (18 items; e.g., "my mum encourages me to watch my weight"/ "my mum sends a strong message to me that I should always look my best"). Items are rated on a 4-point scale (agree to disagree), with lower scores reflecting greater maternal pressures. Good internal consistency was found among daughters in the current study (Cronbach's $\alpha=.81)$.

Psychosocial outcomes related to body image. Self-esteem was assessed using the Rosenberg Self-Esteem Scale (Rosenberg, 1965), shortened to six items as used and validated 
in Project Eat-III (e.g., "On the whole, I am happy with myself"; Neumark-Sztainer et al., 2007). Each item uses a 4-point scale (strongly disagree to strongly agree), with higher scores reflecting greater self-esteem. Internal consistency in the current study was adequate (Cronbach's $\alpha=.69$ and .80 for mothers and daughters, respectively).

Negative affect was measured with the negative items from the validated Positive and Negative Affect Schedule (10 items; Watson, Clark, \& Tellegen, 1988), with a child version used for daughters (5 items; Ebesutani et al., 2012). Both scales are widely used internationally and have demonstrated good psychometric properties. Participants rate the one-word items describing various emotional states (e.g., 'sad', 'afraid') according to how much they had felt that way during the past few weeks on a 5-point scale (very slightly/not at all to extremely/very much). Internal consistency in the current study was good (Cronbach's $\alpha=.90$ and .82 for mothers and daughters, respectively).

A measure of the wider impact of appearance-related worries on various life domains was assessed using a purpose-built scale. Items assessed the extent to which worries or feeling bad about the way they looked had stopped or held back participants' engagement (or the intention to engage) in activities across social, recreational and educational/vocational domains (10 items; e.g., 'go to school', 'go to a social event', 'do a physical activity/sport') over the previous two weeks. Participants rated each item on a 4-point scale (hasn't stopped me at all to stopped me all the time). The mean item score was used, with lower scores indicating greater life engagement. Previous research has found good internal consistency and test re-test reliability for this measure among adolescents (Diedrichs et al., 2015). Good internal consistency was demonstrated in the current study for both mothers $(\alpha=.88)$ and daughters $(\alpha=.92)$.

Help-seeking behaviours. Both mothers and daughters were asked to indicate in two separate purpose-built items "over the past four weeks, have you sought out sources of 
information or support”' for 'body image' and 'eating problems'. Response options included 'doctor', 'counsellor', 'psychologist', 'support group', or 'websites' (other than the Dove website). Results were scored dichotomously, indicating that participants either had, or had not, engaged in help-seeking behaviour ( $0=$ No support, $1=$ Support sought $)$.

In addition to the above measures, we included extra body image and interpersonal relationship measures not reported here for the purpose of a separate program of research on psychometric scale validation and mother-daughter relationships. We also assessed mothers' feedback on the website. Results from this data will be published separately.

Tailoring Measure. To provide mothers with a tailored pathway through the website content, mothers in the website-tailored condition were asked to select two topics that they would most like to learn about in order to best support their daughter's body image. Mothers were presented with five topics to choose from, which corresponded to the risk factor content themes upon which the website content was developed.

Adherence. Mothers in the website conditions were each provided with a unique link to access the website. This link tracked time spent by each mother on the website, number of visits, and the percentage of the tailored pathway viewed by mothers in the website-tailored condition.

\section{Procedure}

After providing informed consent, mother-daughter dyads attended three in-person data collection sessions (baseline, two-weeks post-exposure, six-weeks follow-up) at a research testing location in their respective towns. During the first testing session (baseline) all mothers and daughters completed standardised self-report questionnaires separately in a controlled setting supervised by research staff. Mothers in the website-tailored condition then completed the tailoring measure. All mothers and daughters then had their height and weight measured in private at their discretion. Mothers in both website conditions viewed the 
website individually using their unique link for 30 minutes in silence under standardised conditions, while their daughters were occupied in another room. Mothers in the websitetailored condition were provided with their individualised pathway of website links to follow. Mothers in the website-unstructured condition were simply instructed to browse the website as they wished.

Following exposure to the website, mothers in both website conditions were encouraged to visit the website at home using their unique link, although at-home viewing was not a requirement of the study. Control mothers did not view the website and were not provided with a website link. All mothers and daughters returned to their respective testing locations two- and six-weeks following baseline to complete post-exposure and follow-up questionnaires under standardised conditions. At 12-month follow-up, participants were recontacted via email and telephone, and were invited to complete the final questionnaire online.

\section{Statistical Analyses}

Intervention analyses were conducted via multilevel models in Stata 14.0. Consistent with the Actor-Partner Interdependence Model described by Kenny (1996), we constructed a dyadic model that simultaneously predicted mothers' and daughters' outcomes while also allowing the residual of the outcomes for each mother-daughter dyad to covary. We tested for the hypothesised differences between conditions by including dummy coded variables representing the website-unstructured $(1,0)$ and website-tailored $(1,0)$ conditions. We simultaneously regressed mothers' and daughters' outcomes on demographics characteristics of both dyad members. There were small but significant correlations between mothers' and daughters' age and BMI with body image and a range of risk factor outcome measures at baseline. Therefore, we tested for the effect of condition on daughters when adjusting for their own and their mothers' BMI and age (and vice versa for mothers), while also allowing 
for any residual covariance in outcomes between dyad members, thus recognizing their nonindependence within the model. A cluster robust option for adjusting standard errors was also applied post-estimation to further account for non-independence. We also included baseline outcome scores as covariates in our model. Therefore, the regression coefficients representing the effect of the website-unstructured and website-tailored conditions tested whether outcomes in these two conditions differed from the control condition when adjusting for their initial baseline level. We also controlled for participants' location using dummy coding. Finally, we analyzed the binary data for help-seeking behaviors using dyadic logistic regression. We used this analytic framework to examine differences between conditions at post, 6-week and 12-month follow-up separately. Two dyads in the control condition were removed from 12-month analyses due to reporting they had viewed the Dove website since the previous assessment at 6-week follow-up.

Missing data ranged from $0-4.7 \%$ across outcomes and time points. However, we estimated all models using full information maximum likelihood and were therefore able to include dyads in the model when the outcome for the daughter but not the mother was missing, or vice-versa. When both mother and daughter did not complete an outcome, they were excluded from that particular analysis. Similarly, listwise deletion was used to deal with cases with missing data on core predictors. Cohen's $d$ effect sizes for between-group comparisons were calculated by dividing the difference in estimated means (condition parameter estimate) by the pooled standard deviation (calculated by converting standard errors of estimated means to standard deviations) at each time point (small effect $d=.20$; medium effect $d=.50$, large effect $d=.80$ ). Note that our estimates of effect size represent the difference between conditions in standard deviation units when adjusting for both covariates, and non-independence between dyads. 


\section{Results}

\section{Participant Recruitment and Flow}

Dyads were recruited in May 2014 and baseline testing occurred in June 2014. Figure 1 outlines participant recruitment and retention. The final sample comprised 235 dyads with daughters aged 11-14 and mothers aged 28-54. See Table 1 baseline demographic characteristics and scores on outcome measures.

\section{Intervention Effects}

Table 2 presents results for outcomes with significant results. See online appendix for non-significant outcomes.

Body Image. As seen in Table 2, for mothers and daughters there was no difference in appearance esteem relative to control for either website condition at any time point. Mothers, however, reported higher appearance esteem in the website-tailored condition relative to the website-unstructured condition at 6-week follow-up $(p=.050, d=.39)$. With respect to weight esteem, mothers reported significantly higher weight esteem in the websitetailored condition relative to control at 6-week follow-up $(p=.036, d=.37)$, although this was not maintained at 12-month follow-up. There were no further effects of condition for mothers or daughters on weight esteem.

Risk factors for poor body image. As seen in Table 2, mothers in the websitetailored condition reported significantly lower internalisation of appearance ideals than those in the website-unstructured condition at 12-month follow-up ( $p=.047, d=-.37)$, although neither were significantly different from control. Mothers in the website-tailored condition reported higher perceived sociocultural appearance pressures than control at 12-month follow-up ( $p=.047, d=.38$ ). There was no further effect of condition on internalisation, sociocultural pressures, appearance comparisons, appearance teasing distress, and appearance conversations among mothers or daughters. 
Dyadic factors. As seen in Table 2, mothers in the website-unstructured and websitetailored conditions reported having more conversations with their daughters about body image than control mothers at post-exposure $(p=.001, d=.60$, and $p=.002, d=.58$, respectively) and 6-week follow-up ( $p=.019, d=.41$, and $p=.019, d=.42$, respectively). This effect was not present at 12-month follow-up. Condition did not significantly impact on daughters' reported conversations or perceived maternal pressure.

Psychosocial outcomes. As seen in Table 2, condition did not impact on mothers' negative affect at post-exposure or 6-week follow-up. However, by 12-month follow-up, mothers in the website-tailored condition reported lower negative affect than both control $(p$ $=.033, d=-.52)$ and website-unstructured conditions $(p=.045, d=-.42)$. Daughters in the website-unstructured and website-tailored conditions reported significantly less negative affect than the control condition at 6-week follow-up $(p=.034, d=-.35$, and $p=.045, d=-$ .36 , respectively), not maintained at 12-month follow-up. Regarding self-esteem, mothers in the website-tailored condition reported higher self-esteem than control mothers at postexposure $(p=.010, d=.45)$, and higher self-esteem relative to the website-unstructured condition at 6 -week and 12-month follow-up $(p=.032, d=.37$, and $p=.008, d=.53$, respectively). Condition did not impact on daughters' self-esteem at post-exposure, but daughters in both website-unstructured and website-tailored conditions reported significantly higher self-esteem than control daughters at 6-week follow-up $(p=.031, d=.38$ and $p=$ $.024, d=.40$ respectively), not maintained at 12-month follow-up. Finally, daughters in the website-unstructured condition reported less life engagement than daughters in the control condition at 12-month follow-up $(p=.010, d=.50)$.

Help-seeking behaviours. As seen in Table 2, mothers in the website-tailored condition were over three times more likely than mothers in the control to report that they had sought additional support for body image issues at post-exposure $(p=.004)$. At 6 - week 
follow-up, mothers in the website-unstructured condition were almost three and a half times more likely than control mothers to report that they had sought help for body image ( $p=$ $.005)$, while mothers in the website-tailored condition were close to five times more likely than control mothers to report seeking help for body image $(p<.001)$. By 12-month followup, mothers in the website-tailored condition were still almost three times as likely than control mothers $(p=.049)$, and just over two and a half times more likely than mothers in the website-unstructured condition $(p=.047)$ to report that they had sought additional support around body image.

\section{Adherence}

In addition to 30 minutes of website viewing at baseline, $40.7 \%$ of mothers in the website-unstructured and $68.9 \%$ of mothers in the website-tailored conditions viewed the website at home between baseline and post-intervention testing. Mothers in the tailoredwebsite condition viewed the website for significantly longer at home ( $M=31.85$ minutes; $S D=43.05)$ compare to mothers in the website-unstructured condition $(M=6.38, S D=7.89$; $p=.009)$. In the website-tailored condition, $80 \%$ of mothers viewed every article assigned in their tailored pathway. A further $11 \%$ of mothers viewed $80 \%$ of articles in their pathway, while $9 \%$ viewed $<80 \%$.

\section{Discussion}

For mothers, viewing the Dove Self-Esteem Project Website for Parents with a tailored pathway resulted in significantly higher self-esteem at post-exposure, significantly higher weight esteem at 6-week follow-up, and significantly reduced negative affect at 12month follow-up, relative to the control group. Mothers who viewed the website, either unstructured or via a tailored pathway, also reported having significantly more conversations with their daughters about body image, and were approximately 2.64 - 4.66 times more likely to report that they had sought additional help for body image from health professionals, other 
websites, and/or support groups at post-exposure and 6-week follow-up. Finally, mothers in the tailored-website condition were also 2.64 times more likely than control mothers to report that they had sought additional help for body image at 12-month follow-up. Collectively these results are promising for a brief, low-intensity intervention. They suggest that viewing the website for approximately 30-60 minutes can in the short-term improve mothers' body image and self-esteem, and encourage them to discuss body image with their daughters, and in the longer term it can reduce their levels of negative affect. Considering the intention of this website is to offer a 'first-step' approach, it is also encouraging that viewing the website prompted mothers to seek additional body image support up to 12-months later.

The pattern of results for daughters also suggests that mothers' viewing of the website had a delayed short-term benefit on psychosocial well-being among their daughters. Specifically, daughters whose mothers viewed the website reported significantly improved self-esteem and reduced negative affect at 6-weeks follow-up. The fact that these improvements emerged only at 6-week follow-up suggests that because the intervention is delivered directly to mothers, it may take time for the intervention to diffuse and be of benefit to daughters. Consequently, enhancing the website's capabilities and content in future to increase the ease and frequency with which mothers can share the website's content and key messages with their daughters may help to strengthen and maintain the intervention's effects among daughters (e.g., creating content that mothers can easily share via email or social networking websites, which are popular among adolescent girls).

Contrary to our hypotheses, the intervention did not impact on mother's appearance esteem, comparisons, and conversations relative to the control group. Furthermore, the intervention did not have an impact on daughters' body image, related risk and dyadic factors, or help-seeking behaviours at post-exposure or follow-up. Given that the intervention had short-term benefits for girls' self-esteem and mood, it is interesting that there was no 
significant impact on their body image and related risk factors. It may be that the intervention improved girls' general psychosocial well-being because girls felt their mothers communicated more with them about topics that are relevant to their lives, rather than through other mechanisms related specifically to body image improvement (e.g., increased media literacy). It will be important for future research to investigate the mechanisms by which the website works to improve psychosocial well-being among mothers and their daughters, and to improve body image among mothers. Understanding these mechanisms could inform further optimisation of the website, in order to improve the strength and breadth of positive outcomes.

Another explanation for the unexpected null effects on body image and risk factors for daughters, and the null effects on some outcomes for mothers, may be a lack of statistical power. Specifically, a power analysis indicated that the current sample size was underpowered to detect significance of small effects. Small effect sizes may be expected due to the low-intensity and diffuse nature of this intervention, and the universal sample in the current study. Indeed, consistent with other body image interventions for children delivered to parents (e.g., Corning et al., 2010) and those delivered to universal samples (e.g., Sharpe, Schober, Treasure, \& Schmidt, 2013), our significant effects were small-medium in size ( $d s$ $.35-.55)$. Notably, observed improvements on several outcomes with small effect sizes $(d s$ $.31-.32$ ) failed to reach significance. Future research with a larger sample size may strengthen the pattern of significant findings and is recommended alongside an investigation of the clinical significance of any statistically significant effects.

It may also be the case that this low-intensity intervention would benefit from being supplemented with more targeted, in-depth interventions, in order to achieve consistent significant improvements with larger effect sizes on body image and related risk factor outcomes. If this is the case, it is especially encouraging that in addition to its observed 
benefits for mothers' body image, self-esteem, and negative affect, and girls' psychosocial well-being, mothers who viewed the website were more likely to report that they had sought further help for body image issues. This supports the website's intention of offering a 'firststep' approach, and suggests that the embedded referral strategy to more targeted interventions may be beneficial. In addition to researching supplementary interventions, it may also be beneficial for future research to investigate strategies to increase mothers' engagement with the website and its recommended behaviour change techniques for longer and more frequent periods of time, in an effort to boost intervention effects (e.g., enhancing tailoring mechanisms).

We observed two unexpected findings that warrant attention in future research. The first was that mothers in the tailored-website condition reported significantly greater perceived appearance-related sociocultural pressures at 12-month follow-up relative to the control condition, rather than a reduction in pressures as hypothesised. The research in relation to perceived sociocultural pressures and body image is less well-established with midlife women, however, they have been identified as a risk factor for the development of poor body image among early adult women and girls (Slevec \& Tiggemann, 2011). This effect may be explained by the fact that a large proportion of the website content focuses on helping mothers to identify and challenge appearance pressures in their own, and their daughters', lives from the media, peers, and family. It is important that further research be conducted to explore this unexpected effect, and to measure both awareness and perceived outcomes of these pressures, in order to determine if this is a true iatrogenic effect. The second unexpected finding was that at 12-month follow-up daughters whose mothers viewed the unstructured-website reported poorer life engagement than control daughters. This effect was not present in the website-tailored condition or at any other time point. Despite demonstrating good internal consistency in the current study and one previous study 
(Diedrichs et al., 2015), the life engagement measure has not been well-validated. Therefore, future research should look to replicate and examine this unexpected and possibly iatrogenic effect, and the psychometrics of this scale further.

In this study, we also aimed to investigate if providing mothers with a tailored pathway through the website based upon their preferences could strengthen intervention effects. The overall pattern of results suggests that tailoring was beneficial for mothers. For example, weight esteem, self-esteem, and negative affect improvements were found among mothers in the website-tailored condition only. Benefits at 12-month follow-up among mothers were also observed only in the website-tailored condition. Finally, planned contrasts across the 6-week and 12-month follow-up data revealed that mothers in the tailored-website condition performed significantly better on appearance esteem, self-esteem, internalisation, negative affect, and self-reported help-seeking behaviours than mothers in the websiteunstructured condition. This is consistent with emerging research suggesting that the tailoring of internet-based interventions strengthens their effects (Bennett \& Glasgow, 2009). Future research and development of the website to enhance its tailoring capabilities may be another fruitful avenue for enhancing its benefits for mothers and their daughters.

This study makes an important contribution to existing knowledge on the benefits of accessible and scalable body image interventions for children delivered to parents. The multisite community-based dyadic sample, assessment of child and parent outcomes, and assessment of intervention adherence addresses the methodological limitations of previous studies in this field. Nevertheless, there are several limitations that should be noted. The first limitation is that mothers' exposure to the website occurred in a controlled, standardised setting. While this is useful for the internal validity of the study, it may not reflect the reality of how women consume the internet in their everyday lives, whereby they may be multitasking and/or browsing the internet in smaller chunks of time. Therefore, it is possible that 
this study presents a 'best case scenario' of the intervention effects. Relatedly, mothers and daughters were offered a monetary incentive to participate in this study, which may have increased mothers' willingness to engage with the website. Future research in more naturalistic settings would further enhance the evidence-base for this novel intervention. There was attrition at 12-month follow-up as some mothers were uncontactable. Of the mothers that could be contacted, however, $100 \%$ consented and took part. In terms of the analyses, multiple tests were conducted and thus it must be acknowledged that some of our significant findings could be the result of chance. Future research should aim to replicate our findings, ideally with a larger sample. Finally, as previously mentioned, the life engagement measure requires further psychometric validation.

\section{Conclusions}

Considering that the observed benefits for mothers' and daughters' psychosocial wellbeing emerged after mothers' very brief exposure to a website (i.e., 30-60 minutes on average), the website's potential as a convenient 'first-step' intervention for parents is promising. Despite the observed effect sizes being predominantly small in size, the scalability of this intervention and its existing reach (i.e., an average of 792,000 visits per year across multiple countries) is encouraging given that when interventions with small effects are delivered at scale they have the potential to provide population benefits (Kazdin \& Blase, 2011; Rose, 1985). Future research to improve the mechanisms by which this website can confer sustained benefits for body image and psychosocial outcomes, and to explore the added benefits of supplementary in-depth, targeted interventions, would be welcome. In conclusion, this study suggests that this novel, low-intensity, scalable intervention may offer a useful first-step towards improving mothers' body image, self-esteem, negative affect and related help-seeking behaviours, increasing conversations about body image among mothers and their daughters, and to improving girls' self-esteem and negative affect. 


\section{References}

Al Sabbah, H., Vereecken, C. A., Elgar, F. J., Nansel, T., Aasvee, K., Abdeen, Z., . . Maes, L. (2009). Body weight dissatisfaction and communication with parents among adolescents in 24 countries: international cross-sectional survey. BMC Public Health, 9, 52. doi:10.1186/1471-2458-9-52

Atkinson, M. J., \& Wade, T. D. (2013). Enhancing dissemination in selective eating disorders prevention: An investigation of voluntary participation among female university students. Behaviour Research and Therapy, 51, 806-816. doi:10.1016/j.brat.2013.09.012

Bennett, G. G., \& Glasgow, R. E. (2009). The delivery of public health interventions via the internet: Actualizing their potential. Annual Review of Public Health, 30, 273-292. doi:10.1146/annurev.publhealth.031308.100235

Corning, A. F., Gondoli, D. M., Bucchianeri, M. M., \& Salafia, E. H. B. (2010). Preventing the development of body issues in adolescent girls through intervention with their mothers. Body Image, 7, 289-295.

Diedrichs, P. C., Atkinson, M. J., Steer, R. J., Garbett, K. M., Rumsey, N., \& Halliwell, E. (2015). Effectiveness of a brief school-based body image intervention 'Dove Confident Me: Single Session' when delivered by teachers and researchers: Results from a cluster randomised controlled trial. Behaviour Research and Therapy. doi:10.1016/j.brat.2015.09.004

Ebesutani, C., Regan, J., Smith, A., Reise, S., Higa-McMillan, C., \& Chorpita, B. F. (2012). The 10-Item Positive and Negative Affect Schedule for Children, Child and Parent Shortened Versions: Application of Item Response Theory for More Efficient Assessment. J Psychopathol Behav Assess, 34, 191-203. 
Fairburn, C. G., \& Patel, V. (2014). The global dissemination of psychological treatments: A road map for research and practice. American Journal of Psychiatry, 171, 495-498. doi:0.1176/appi.ajp.2013.13111546

Hart, L. M., Cornell, C., Damiano, S. R., \& Paxton, S. J. (2014). Parents and prevention: A systematic review of interventions involving parents that aim to prevent body dissatisfaction or eating disorders. International Journal of Eating Disorders, 48, 157169. doi:10.1002/eat.22284

Ingoldsby, E. M. (2010). Review of interventions to improve family engagement and retention in parent and child mental health programs. Journal of Child and Family Studies, 19, 629-645. doi:0.1007/s10826-009-9350-2

Jacobi, C., \& Fittig, E. (2010). Psychosocial risk factors for eating disorders. In S. W. Agras (Ed.), The Oxford Handbook of Eating Disorders (pp. 123-136). New York: Oxford University Press.

Jones, D. C. (2001). Social comparison and body image: Attractiveness comaprisons to models and peers among adolescent girls and boys. Sex Roles, 45, 645-664. doi:10.1023/A:1014815725852

Jones, D. C., Vigfusdottir, T. H., \& Lee, Y. (2004). Body image and appearance culture among adolescent girls and boys: An examination of friend conversations, peer criticism, appearance magazines and the internalisation of appearance ideals. Journal of Adolescent Research, 19, 323-339. doi:10.1177/0743558403258847

Kazdin, A. E., \& Blase, S. L. (2011). Rebooting psychotherapy research and pracice to reduce the burden of mental illness. Perspectives on Psychological Science, 61, 2137. doi:10.1177/1745691610393527

Kenny, D. A. (1996). Models of nonindependence in dyadic research. Journal of Social and Personal Relationships, 13, 279-294. 
Kenny, D. A., Kashy, D. A., \& Cook, W. L. (2006). Dyadic data analysis. New York: Guilford Press.

Kilpela, L. S., Hill, K., Kelly, M. C., Elmquist, J., Ottoson, P., Keith, D., . . Becker, C. B. (2014). Reducing eating disorder risk factors: A controlled investigation of a blended task-shifting/train-the-trainer approach to dissemination and implementation. Behaviour Research and Therapy, 63, 70-82. doi:10.1016/j.brat.2014.09.005

Mendelson, B. K., Mendelson, M. J., \& White, D. R. (2001). Body-Esteem Scale for Adolescents and Adults. Journal of Personality Assessment, 76(1), 90 - 106. Retrieved from http://www.informaworld.com/10.1207/S15327752JPA7601_6

Mendelson, B. K., White, D. R., \& Mendelson, M. J. (1997) Manual for the body-esteem scale for adolescents and adults. Vol. 16 (2). Research Bulletin. Montreal: Concordia University.

Neumark-Sztainer, D., Paxton, S. J., Hannan, P. J., Haines, J., \& Story, M. (2006). Does Body Satisfaction Matter? Five-year Longitudinal Associations between Body Satisfaction and Health Behaviors in Adolescent Females and Males. Journal of Adolescent Health, 39(2), 244-251. doi:10.1016/j.jadohealth.2005.12.001

Neumark-Sztainer, D., Wall, M., Haines, J., Story, M., Sherwood, N. E., \& Van den Berg, P. (2007). Shared risk and protective factors for overweight and disordered eating in adolescents. Journal of Preventive Medicine, 33, 359-369.

Patel, V., Kieling, C., Maulik, P. K., \& Divan, G. (2013). Improving access to care for children with mental disorders: a global perspective. Archives of Disease in Childhood, 98, 323-327. doi:10.1136/archdischild-2012-302079

Paxton, S. J. (2015). Social policy and prevention. In L. Smolak \& M. P. Levine (Eds.), The Wiley Handbook of Eating Disorders. Chichester, UK: John Wiley \& Sons, Ltd. 
Rodgers, R. F., \& Chabrol, H. (2009). Parental attitudes, body image disturbance and disordered eating amongst adolescents and young adults: A review. European Eating Disorders Review, 17, 137-151. doi:10.1002/erv.907

Rose, G. (1985). Sick individuals and sick populations. International Journal of Epidemiology, 14, 32-38.

Rosenberg, M. (1965). Society and the adolescent self-image. Princeton, NJ: Princeton University Press.

Sharpe, H., Schober, I., Treasure, J., \& Schmidt, U. (2013). Feasibility, acceptability and efficacy of a school-based prevention programme for eating disorders: cluster randomised controlled trial. British Journal of Psychiatry, 203, 428-435. doi:10.1192/bjp.bp.113.128199

Slevec, J. H., \& Tiggemann, M. (2011). Predictors of body dissatisfaction and disordered eating in middle-age women. Clinical Psychology Review, 31, 515-524. doi:10.1016/j.cpr.2010.12.002

Sniezek, R. (2006). Parental criticism and eating disturbance in adolescent females: Implications for a multifaceted eating disorder prevention program. Dissertation Abstracts International, 67, 3467.

Stice, E., \& Bearman, S. K. (2001). Body-image and eating disturbances prospectively predict increases in depressive symptoms in adolescent girls: A growth curve analysis. Developmental Psychology, 37, 597-607.

Stice, E., Becker, C. B., \& Yokum, S. (2013). Eating disorder prevention: Current evidencebased and future directions. International Journal of Eating Disorders, 46, 478-485. doi:10.1002/eat.22105 
Taylor, C. B., Bryson, S., Luce, K. H., Cunning, D., Celio Doyle, A., Abascal, L. B., . . . Wilfley, D. E. (2006). Prevention of eating disorders in at-risk collage-age women. Archives of General Psychiatry, 63, 881-888. doi:10.1001/archpsyc.63.8.881

Thompson, J. K., van den Berg, P., Roehrig, M., Guarda, A. S., \& Heinberg, L. J. (2004). The Sociocultural Attitudes Towards Appearance Scale-3 (SATAQ-3): Development and validation. International Journal of Eating Disorders, 35, 293-304.

Twisk, J. W. R. (2006). Applied multilevel analysis. UK: Cambridge University Press.

Watson, D., Clark, K., A, \& Tellegen, A. (1988). Development and validation of brief measures of positive and negative affect: the PANAS scales. Journal of Personality and Social Psychology, 6, 1063-1070. 


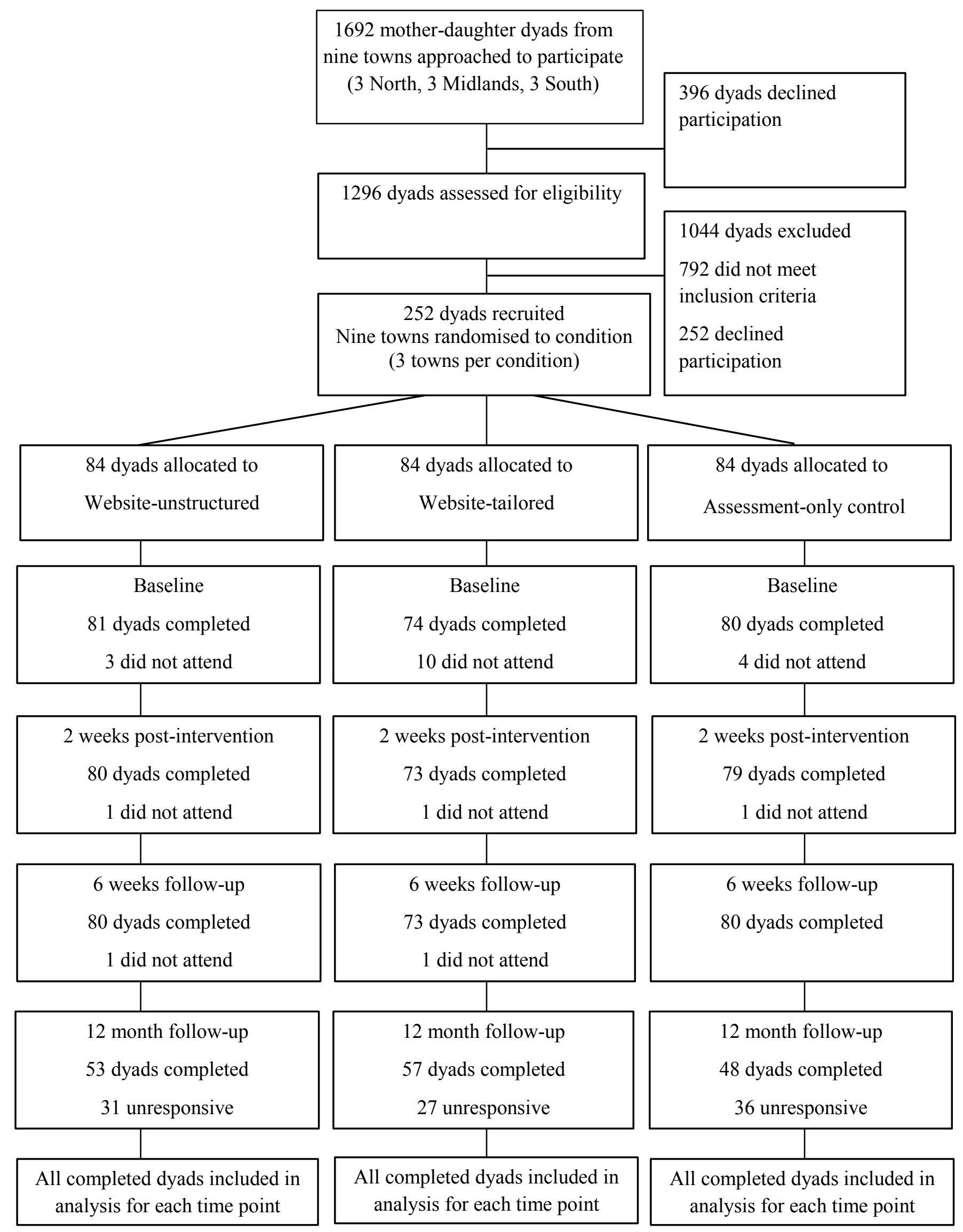

Figure 1. CONSORT diagram of participant flow. 
Table 1

Baseline demographic characteristics and scores on outcome measures (means and standard deviations, unless otherwise indicated)

\begin{tabular}{|c|c|c|c|c|c|c|}
\hline & \multicolumn{3}{|c|}{ Mothers $(N=235)$} & \multicolumn{3}{|c|}{ Daughters $(N=235)$} \\
\hline & $\begin{array}{c}\text { Control } \\
n=80\end{array}$ & $\begin{array}{c}\text { Website- } \\
\text { unstructured } \\
n=81\end{array}$ & $\begin{array}{c}\text { Website- } \\
\text { tailored } \\
n=74 \\
\end{array}$ & $\begin{array}{c}\text { Control } \\
n=80\end{array}$ & $\begin{array}{c}\text { Website- } \\
\text { unstructured } \\
n=81\end{array}$ & $\begin{array}{c}\text { Website- } \\
\text { tailored } \\
n=74\end{array}$ \\
\hline$\overline{\text { Age }}$ & $41.33(5.30)$ & $42.26(5.40)$ & $41.97(4.78)$ & $12.99(0.86)$ & $12.98(0.82)$ & $13.03(0.89)$ \\
\hline Body Mass Index & $27.50(6.04)$ & $25.72(4.63)$ & $28.96(6.30)$ & $20.56(3.87)$ & $20.25(4.75)$ & $22.13(4.50)$ \\
\hline Born in the UK $(n, \%)$ & $80(100)$ & $81(100)$ & $71(92.31)$ & $80(100)$ & $81(100)$ & $73(98.6)$ \\
\hline \multicolumn{7}{|l|}{ Marital Status (n, \%) } \\
\hline Single & $12(15)$ & $3(3.7)$ & $7(9.5)$ & - & - & - \\
\hline Cohabiting & $1(1.3)$ & $7(8.6)$ & $4(5.4)$ & - & - & - \\
\hline Married & $57(71.3)$ & $55(67.9)$ & $58(78.4)$ & - & - & - \\
\hline Divorced & $10(12.5)$ & $13(16.0)$ & $4(5.4)$ & - & - & - \\
\hline Widowed & 0 & $3(3.7)$ & $1(1.4)$ & - & - & - \\
\hline \multicolumn{7}{|l|}{ Ethnicity $(n, \%)$} \\
\hline White & $75(93.8)$ & $75(92.6)$ & $69(93.0)$ & $73(92.3)$ & $72(88.9)$ & $66(89.1)$ \\
\hline Other & $5(6.2)$ & $6(7.4)$ & $5(7.0)$ & $7(8.7)$ & $9(11.1)$ & $8(10.9)$ \\
\hline Social Grade $(n, \%)$ & & & & - & - & - \\
\hline $\begin{array}{l}\text { A \& B } \\
\text { (higher/ intermediate managerial, admin, professional) }\end{array}$ & $8(10)$ & $20(24.7)$ & $16(21.6)$ & - & - & - \\
\hline
\end{tabular}




\section{$\mathrm{C} 1 \& \mathrm{C} 2$}

(supervisory/ junior managerial, admin, professional;

skilled manual worker)

$\mathrm{D} \& \mathrm{E}$

(semi-skilled/ unskilled manual worker; pensioner,

retired, unemployed)

Appearance esteem

Weight esteem

Internalisation of appearance ideals

Sociocultural pressures

Appearance comparisons

Appearance teasing distress

Appearance conversations

Frequency of mother/daughter body image conversations

Perceived maternal pressures

Negative affect

Self-esteem

Life engagement

Help-seeking behaviours ( $n, \%)$

\begin{tabular}{|c|c|c|c|c|c|}
\hline $60(75)$ & $57(70.4)$ & $39(52.7)$ & - & - & - \\
\hline $12(15)$ & $4(4.9)$ & $19(25.7)$ & - & - & - \\
\hline $3.10(0.64)$ & $3.27(0.61)$ & $3.26(0.62)$ & $3.29(0.82)$ & $3.43(0.56)$ & $3.11(0.85)$ \\
\hline $2.69(0.83)$ & $2.84(0.79)$ & $2.70(0.81)$ & $3.24(1.00)$ & $3.44(0.86)$ & $3.06(1.02)$ \\
\hline $2.45(0.99)$ & $2.49(0.93)$ & $2.26(0.96)$ & $2.50(0.99)$ & $2.41(0.79)$ & $2.69(1.20)$ \\
\hline $1.64(0.66)$ & $1.55(0.61)$ & $1.43(0.59)$ & $1.57(0.59)$ & $1.37(0.42)$ & $1.59(0.67)$ \\
\hline $2.92(0.88)$ & $2.91(0.97)$ & $2.63(0.85)$ & $2.22(0.82)$ & $2.15(0.78)$ & $2.30(0.99)$ \\
\hline- & - & - & $1.75(1.09)$ & $1.54(1.00)$ & $1.98(1.22)$ \\
\hline $3.30(1.15)$ & $3.25(0.98)$ & $3.31(0.95)$ & $2.65(1.21)$ & $2.41(1.05)$ & $2.60(1.26)$ \\
\hline $3.79(0.96)$ & $3.63(0.94)$ & $3.88(0.83)$ & $2.58(1.08)$ & $2.67(1.04)$ & $2.62(1.11)$ \\
\hline - & - & - & $3.21(0.45)$ & $3.16(0.44)$ & $3.13(0.47)$ \\
\hline $2.83(0.55)$ & $2.78(0.52)$ & $2.86(0.45)$ & $1.84(0.86)$ & $1.86(0.75)$ & $1.98(0.94)$ \\
\hline $2.86(0.45)$ & $2.99(0.46)$ & $3.05(0.54)$ & $2.74(0.67)$ & $2.81(0.5)$ & $2.57(0.65)$ \\
\hline $1.51(0.57)$ & $1.29(0.4)$ & $1.30(0.43)$ & $1.39(0.54)$ & $1.25(0.36)$ & $1.37(0.50)$ \\
\hline $45(56)$ & $36(44)$ & $38(51)$ & $30(38)$ & $36(44)$ & $32(43)$ \\
\hline
\end{tabular}


Table 2

Estimated means and standard errors by condition and time, and between-condition pairwise comparisons at each time point

\begin{tabular}{|c|c|c|c|c|c|c|c|c|c|c|c|c|}
\hline \multirow[b]{2}{*}{$\begin{array}{l}\text { Measure } \\
\text { (baseline } \\
\text { covariate) }\end{array}$} & \multirow{2}{*}{$\begin{array}{c}\text { Control } \\
M(S E)\end{array}$} & \multirow{2}{*}{$\begin{array}{c}\text { Website } \\
M(S E)\end{array}$} & \multirow{2}{*}{$\begin{array}{c}\text { Tailored } \\
M(S E)\end{array}$} & \multicolumn{3}{|c|}{ Website vs Control } & \multicolumn{3}{|c|}{ Tailored vs Control } & \multicolumn{3}{|c|}{ Tailored vs Website } \\
\hline & & & & $b(S E)$ & $95 \% C I$ & $p(d)$ & $b(S E)$ & $95 \% C I$ & $p(d)$ & $b(S E)$ & $95 \% C I$ & $p(d)$ \\
\hline \multicolumn{13}{|c|}{ Appearance esteem } \\
\hline \multicolumn{13}{|c|}{ Mothers (3.25) } \\
\hline Post & $3.24(0.05)$ & $3.30(0.03)$ & $3.36(0.04)$ & $0.05(0.06)$ & $-0.06-0.16$ & $.347(.15)$ & $0.12(0.07)$ & $-0.01-0.25$ & $.075(.33)$ & $0.07(0.06)$ & $-0.05-0.18$ & $.251(.25)$ \\
\hline 6-week & $3.31(0.06)$ & $3.28(0.04)$ & $3.42(0.05)$ & $-0.03(0.06)$ & $-0.15-0.10$ & $.678(-.07)$ & $0.12(0.09)$ & $-0.05-0.29$ & $.180(.27)$ & $0.14(0.07)$ & $0.00-0.28$ & $.050(.39)$ \\
\hline 12-month & $3.31(0.07)$ & $3.27(0.06)$ & $3.26(0.06)$ & $-0.04(0.09)$ & $-0.21-0.13$ & $.641(-.09)$ & $-0.05(0.09)$ & $-0.22-0.13$ & $.612(-.12)$ & $-0.01(0.09)$ & $-0.18-0.17$ & $.955(-.02)$ \\
\hline \multicolumn{13}{|c|}{ Weight esteem } \\
\hline \multicolumn{13}{|c|}{ Mothers (2.80) } \\
\hline Post & $2.89(0.06)$ & $2.88(0.04)$ & $2.93(0.05)$ & $-0.01(0.07)$ & $-0.14-0.12$ & $.894(-.02)$ & $0.04(0.08)$ & $-0.11-0.19$ & $.608(.09)$ & $0.05(0.06)$ & $-0.08-0.17$ & $.445(.14)$ \\
\hline 6-week & $2.91(0.05)$ & $2.97(0.04)$ & $3.07(0.06)$ & $0.06(0.06)$ & $-0.06-0.18$ & $.337(.16)$ & $0.16(0.08)$ & $0.01-0.31$ & $.036(.37)$ & $0.10(0.07)$ & $-0.04-0.24$ & $.145(.25)$ \\
\hline 12-month & $2.89(0.07)$ & $2.89(0.07)$ & $2.89(0.08)$ & $-0.01(0.09)$ & $-0.19-0.18$ & $.953(-.02)$ & $0.00(0.11)$ & $-0.21-0.20$ & $.966(.00)$ & $0.00(0.11)$ & $-0.21-0.21$ & $.992(.00)$ \\
\hline \multicolumn{13}{|c|}{ Internalisation of appearance ideals } \\
\hline \multicolumn{13}{|c|}{ Mothers (2.41) } \\
\hline Post & $2.15(0.09)$ & $2.16(0.08)$ & $2.16(0.10)$ & $0.01(0.11)$ & $-0.21-0.24$ & $.913(.01)$ & $0.01(0.13)$ & $-0.25-0.26$ & $.957(.01)$ & $-0.01(0.13)$ & $-0.25-0.24$ & $.964(-.01)$ \\
\hline 6-week & $1.99(0.07)$ & $2.05(0.07)$ & $1.91(0.09)$ & $0.06(0.1)$ & $-0.14-0.26$ & $.542(.10)$ & $-0.08(0.12)$ & $-0.31-0.15$ & $.486(-.12)$ & $-0.14(0.12)$ & $-0.38-0.09$ & $.228(-.22)$ \\
\hline 12-month & $1.95(0.08)$ & $2.13(0.1)$ & $1.87(0.09)$ & $0.18(0.13)$ & $-0.07-0.44$ & $.163(.27)$ & $-0.08(0.12)$ & $-0.31-0.15$ & $.474(-.14)$ & $-0.26(0.13)$ & $-0.53-0.00$ & $.047(-.37)$ \\
\hline
\end{tabular}




\begin{tabular}{|c|c|c|c|c|c|c|c|c|c|c|c|c|}
\hline \multicolumn{13}{|c|}{ Perceived sociocultural pressures } \\
\hline \multicolumn{13}{|c|}{ Mothers (1.49) } \\
\hline Post & $1.49(0.05)$ & $1.53(0.05)$ & $1.50(0.05)$ & $0.04(0.07)$ & $-0.11-0.18$ & $.627(.10)$ & $0.01(0.07)$ & $-0.13-0.15$ & $.918(.03)$ & $-0.03(0.07)$ & $-0.16-0.10$ & $.667(-.08)$ \\
\hline 6-week & $1.46(0.06)$ & $1.48(0.04)$ & $1.44(0.05)$ & $0.02(0.07)$ & $-0.13-0.17$ & $.790(.05)$ & $-0.02(0.07)$ & $-0.16-0.13$ & $.811(-.05)$ & $-0.04(0.07)$ & $-0.17-0.09$ & $.572(-.11)$ \\
\hline 12-month & $1.38(0.05)$ & $1.52(0.06)$ & $1.63(0.12)$ & $0.14(0.08)$ & $-0.01-0.29$ & $.073(.37)$ & $0.25(0.12)$ & $0.00-0.49$ & $.047(.38)$ & $0.11(0.13)$ & $-0.15-0.36$ & $.403(.17)$ \\
\hline \multicolumn{13}{|c|}{ Frequency of mother/daughter conversations } \\
\hline \multicolumn{13}{|c|}{ Mothers (3.71) } \\
\hline Post & $2.84(0.12)$ & $3.37(0.10)$ & $3.38(0.12)$ & $0.54(0.16)$ & $0.23-0.85$ & $.001(.60)$ & $0.55(0.17)$ & $0.21-0.88$ & $.002(.58)$ & $0.01(0.16)$ & $-0.31-0.32$ & $.971(.01)$ \\
\hline 6-week & $3.07(0.11)$ & $3.44(0.11)$ & $3.44(0.11)$ & $0.37(0.16)$ & 0.06-0.69 & $.019(.41)$ & $0.37(0.16)$ & $0.06-0.68$ & $.019(.42)$ & $0.00(0.16)$ & $-0.32-0.31$ & $.985(.00)$ \\
\hline 12-month & $3.44(0.12)$ & $3.44(0.13)$ & $3.61(0.09)$ & $0.00(0.18)$ & $-0.35-0.35$ & $.999(.00)$ & $0.17(0.15)$ & $-0.13-0.47$ & $.268(.25)$ & $0.17(0.16)$ & $-0.15-0.49$ & $.294(.20)$ \\
\hline \multicolumn{13}{|c|}{ Negative affect } \\
\hline \multicolumn{13}{|c|}{ Mothers (2.81) } \\
\hline Post & $2.64(0.05)$ & $2.67(0.05)$ & $2.70(0.04)$ & $0.03(0.07)$ & $-0.11-0.17$ & $.669(.07)$ & $0.06(0.07)$ & $-0.07-0.19$ & $.360(.17)$ & $0.03(0.07)$ & $-0.10-0.16$ & $.639(.08)$ \\
\hline 6-week & $2.80(0.05)$ & $2.73(0.05)$ & $2.79(0.05)$ & $-0.06(0.07)$ & $-0.20-0.08$ & $.404(-.15)$ & $-0.01(0.07)$ & $-0.14-0.13$ & $.923(-.03)$ & $0.05(0.07)$ & $-0.09-0.20$ & $.467(.12)$ \\
\hline 12-month & $2.74(0.06)$ & $2.73(0.06)$ & $2.56(0.05)$ & $-0.02(0.09)$ & $-0.19-0.16$ & $.842(-.05)$ & $-0.19(0.09)$ & $-0.36--0.02$ & $.033(-.52)$ & $-0.17(0.08)$ & $-0.33-0.00$ & $.045(-.42)$ \\
\hline \multicolumn{13}{|c|}{ Daughters (1.84) } \\
\hline Post & $1.86(0.08)$ & $1.84(0.09)$ & $1.82(0.08)$ & $-0.02(0.12)$ & $-0.25-0.21$ & $.856(-.03)$ & $-0.05(0.12)$ & $-0.27-0.18$ & $.694(-.08)$ & $-0.02(0.13)$ & $-0.27-0.22$ & $.844(-.03)$ \\
\hline 6-week & $1.97(0.10)$ & $1.71(0.08)$ & $1.73(0.07)$ & $-0.26(0.12)$ & $-0.51--0.02$ & $.034(-.35)$ & $-0.25(0.12)$ & $-0.49--0.01$ & $.045(-.36)$ & $0.02(0.12)$ & $-0.21-0.25$ & $.880(.03)$ \\
\hline 12-month & $2.13(0.13)$ & $2.13(0.10)$ & $1.88(0.11)$ & $0.00(0.17)$ & $-0.33-0.33$ & $.996(.00)$ & $-0.25(0.17)$ & $-0.58-0.07$ & $.129(-.32)$ & $-0.26(0.16)$ & $-0.56-0.05$ & $.099(-.35)$ \\
\hline
\end{tabular}




\begin{tabular}{|c|c|c|c|c|c|c|c|c|c|c|c|c|}
\hline \multicolumn{13}{|l|}{ Self-esteem } \\
\hline \multicolumn{13}{|c|}{ Mothers (2.99) } \\
\hline Post & $2.91(0.04)$ & $2.99(0.04)$ & $3.07(0.05)$ & $0.08(0.05)$ & $-0.02-0.18$ & $.102(.24)$ & $0.16(0.06)$ & $0.04-0.29$ & $.010(.45)$ & $0.08(0.06)$ & $-0.05-0.2$ & $.221(.22)$ \\
\hline 6-week & $2.99(0.05)$ & $2.97(0.04)$ & $3.13(0.06)$ & $-0.02(0.06)$ & $-0.14-0.1$ & $.802(-.05)$ & $0.14(0.08)$ & $-0.01-0.29$ & $.069(.32)$ & $0.15(0.07)$ & 0.01-0.29 & $.032(.37)$ \\
\hline 12-month & $2.97(0.05)$ & $2.87(0.05)$ & $3.10(0.07)$ & $-0.09(0.07)$ & $-0.24-0.05$ & $.197(-.26)$ & $0.13(0.09)$ & $-0.04-0.3$ & $.131(.30)$ & $0.23(0.08)$ & $0.06-0.39$ & $.008(.53)$ \\
\hline \multicolumn{13}{|c|}{ Daughters (2.75) } \\
\hline Post & $2.83(0.05)$ & $2.87(0.04)$ & $2.89(0.05)$ & $0.04(0.06)$ & $-0.09-0.16$ & $.564(.11)$ & $0.06(0.07)$ & $-0.08-0.2$ & $.418(.15)$ & $0.02(0.07)$ & $-0.11-0.15$ & $.725(.06)$ \\
\hline 6-week & $2.82(0.05)$ & $2.95(0.04)$ & $2.98(0.05)$ & $0.14(0.06)$ & 0.01-0.26 & $.031(.38)$ & $0.16(0.07)$ & $0.02-0.3$ & $.024(.40)$ & $0.03(0.07)$ & $-0.1-0.16$ & $.698(.08)$ \\
\hline 12-month & $2.78(0.08)$ & $2.83(0.07)$ & $2.83(0.06)$ & $0.05(0.10)$ & $-0.14-0.25$ & $.597(.10)$ & $0.05(0.10)$ & $-0.14-0.24$ & $.575(.11)$ & $0(0.09)$ & $-0.18-0.19$ & $.990(.00)$ \\
\hline \multicolumn{13}{|c|}{ Life engagement $^{a}$} \\
\hline \multicolumn{13}{|c|}{ Daughters (1.29) } \\
\hline Post & $1.24(0.04)$ & $1.18(0.03)$ & $1.28(0.04)$ & $-0.06(0.05)$ & $-0.15-0.03$ & $.204(-.21)$ & $0.04(0.05)$ & $-0.06-0.14$ & $.440(.13)$ & $0.10(0.05)$ & $0.00-0.20$ & $.051(.35)$ \\
\hline 6-week & $1.19(0.03)$ & $1.17(0.03)$ & $1.21(0.03)$ & $-0.03(0.04)$ & $-0.10-0.05$ & $.496(-.12)$ & $0.01(0.05)$ & $-0.08-0.11$ & $.778(.04)$ & $0.04(0.04)$ & $-0.04-0.12$ & $.332(.16)$ \\
\hline 12-month & $1.16(0.03)$ & $1.30(0.05)$ & $1.26(0.05)$ & $0.15(0.06)$ & $0.03-0.26$ & $.010(.50)$ & $0.10(0.06)$ & $-0.02-0.22$ & $.097(.35)$ & $-0.04(0.07)$ & $-0.19-0.10$ & $.549(-.11)$ \\
\hline \multicolumn{13}{|c|}{ Additional help sought for body image } \\
\hline Mothers $(3.2$ & Yes (\%) & Yes $(\%)$ & Yes $(\%)$ & $b(S E)$ & $95 \% C I$ & $p(O R)$ & $b(S E)$ & $95 \% C I$ & $p(O R)$ & $b(S E)$ & $95 \% C I$ & $p(O R)$ \\
\hline Post & $16(24)$ & $23(32)$ & $29(46)$ & $0.57(0.41)$ & $-0.23-1.37$ & $.164(1.77)$ & $1.16(0.4)$ & $0.37-1.96$ & $.004(3.19)$ & $0.6(0.42)$ & $-0.22-1.42$ & $.155(1.82)$ \\
\hline 6-week & $12(18)$ & $24(33)$ & $28(44)$ & $1.24(0.44)$ & $0.37-2.11$ & $.005(3.46)$ & $1.54(0.43)$ & $0.69-2.39$ & $<.001(4.66)$ & $0.3(0.42)$ & $-0.51-1.12$ & $.468(1.35)$ \\
\hline 12-month & $8(12)$ & $8(11)$ & $15(24)$ & $0.12(0.56)$ & $-0.99-1.23$ & $.831(1.13)$ & $1.09(0.55)$ & 0.01-2.17 & $.049(2.97)$ & $0.97(0.49)$ & 0.01-1.92 & $.047(2.64)$ \\
\hline
\end{tabular}

${ }^{a}$ Lower scores indicate greater life engagement 
Key Points:

1) Few evidence-based body image interventions have been widely disseminated, due to an over-reliance on external providers and face-to-face interventions.

2) Empirical evidence shows parents are a key influence on adolescent girls' body image.

3) This study evaluated the impact of brief exposure to a low-intensity, internetbased intervention delivered to mothers among mother-daughter dyads.

4) The intervention had benefits for mothers' body image, self-esteem and helpseeking behaviors, and daughters' self-esteem and negative affect.

5) Results suggest this intervention offers a promising 'first-step' approach to improving mothers' body image and related psychosocial outcomes among their daughters. 